\title{
Epidémie de la panachure jaune du riz: une contrainte biotique majeure de la production rizicole dans les périmètres irrigués du Niger
}

\author{
Souley ISSAKA ${ }^{1 *}$, Mariama Abdoulaye Moussa SOULEY ${ }^{2}$ et Adamou BASSO $^{3}$
}

${ }^{1}$ Faculté des Sciences Agronomiques (FSA), Université Boubakar Bâ de Tillabéri, BP. 175 Tillabéri, Niger. ${ }^{2}$ Ministère de l'Industrie, BP 11700 Niamey, Niger.

${ }^{3}$ Institut National de la Recherche Agronomique du Niger (INRAN), BP 429, Niamey, Niger. *Auteur correspondant ; E-mail: souleymayaki@gmail.comTel : +22799774638/951212 18,

\section{REMERCIEMENTS}

Cette étude a été financée par le Programme de Productivité Agricole en Afrique de l'Ouest (PPAAO)Niger.
Received: $10-06-2021$
Accepted: 07-10-2021
Published: $30-10-2021$

\section{RESUME}

La panachure jaune du riz, causée par le Rice Yellow Mottle Virus (RYMV), est présente au Niger depuis plus de trois décennies, avec des pertes importantes de récoltes. Une réévaluation de cette maladie sur les périmètres irrigués a été faite pour estimer son incidence et les pertes de récoltes qu'elle engendre. L'étude a consisté à: (i) surveiller l'épidémie dans quelques périmètres rizicoles sur deux campagnes, (ii) calculer les pertes de production en grains enregistrées et (ii) caractériser quelques isolats du virus. Elle a révélé que la maladie est toujours présente dans les périmètres irrigués du pays. Son incidence moyenne est de 5 à $30 \%$, en saison sèche et en saison humide, elle varie entre 5 et $70 \%$, avec des niveaux d'infestation variables d'un périmètre à l'autre. Les pertes moyennes de production en grains enregistrées en champ paysan sont variables selon les sites et oscillent entre $27,17 \%$ et $63,26 \%$ en saison humide où lesdites pertes ont été plus importantes. Ces résultats montrent que l'épidémie de la panachure jaune du riz est toujours très fréquente au Niger et constitue une contrainte majeure de la production rizicole, dominée par la prévalence d'isolats de contournement de la résistance et l'utilisation presque exclusive de deux principales variétés de riz que sont IR1529-680-3-1 et Kogoni91-1.

(C) 2021 International Formulae Group. All rights reserved.

Mots clés : panachure jaune du riz, Niger, incidence et pertes de production.

\section{Rice yellow mottle epidemic: a major biotic constraint of rice production in the irrigated perimeters of Niger Republic}

\begin{abstract}
The rice yellow mottle due to Rice Yellow Mottle Virus (RYMV) is present in Niger Republic for more than three decades, with significant crop losses. A reassessment of this disease on irrigated areas has been made, to estimate its incidence and the crop losses it causes. The study consisted of: (i) monitoring the
\end{abstract}


epidemic in a few rice-growing perimeters over two seasons, (ii) calculating the recorded grains production losses and (ii) characterizing some isolates of the virus. It revealed that the disease is still present in the country's rice irrigated perimeters. Its average incidence is $5-30 \%$ in the dry season and in the wet season it varies between 5 and $70 \%$, with varying levels of infestation depending on the perimeter. Average grains production losses recorded in the producer's field vary from site to site and range from $27.17 \%$ to $63.26 \%$ in the wet season when these losses were greater. These results show that the Rice yellow mottling epidemic is still very frequent in Niger Republic and constitutes a major constraint of rice production, dominated by the prevalence of Resistance Breaking isolates $(\mathrm{RB})$ and the almost exclusive use of two main rice varieties which are IR1529-680-3-1 and Kogoni91-1.

(C) 2021 International Formulae Group. All rights reserved.

Keywords: Rice Yellow Mottle Virus (RYMV), Niger, incidence and production losses.

\section{INTRODUCTION}

La panachure jaune du riz est une maladie virale émergente en Afrique, transmise par les insectes et par voie mécanique (Kouassi et al., 2005; Ochola and Tusiime, 2011; Bouet et al., 2012). Elle a été décrite pour la première fois en 1966 au lac Victoria, au Kenya (N'dikumana et al., 2011). La maladie a été par la suite observée dans 28 pays d'Afrique de l'Est, du Centre et dans de nombreux pays d'Afrique de l'Ouest, avec des incidences variables de 17 à $100 \%$ (Soko et al., 2015; Oludare et al., 2016 ; Kam et al., 2017). La maladie a aussi été décrite en Afrique Centrale, principalement au Tchad, au Cameroun et en République Centre Africaine (Traoré et al., 2010; Longué et al., 2016; Longué et al., 2017) ainsi que dans d'autres pays comme l'Ouganda (Fargette et al., 2006 ; Ochola et al., 2015), la Gambie (Séré et al., 2008), le Rwanda et le Burundi (Ndikumana et al., 2011 ; Ndikumana et al., 2012). Récemment encore, la présence de la panachure jaune du riz a été rapportée au Sénégal, jusque-là considéré comme indemne (Tall et al., 2020). C'est dire donc que la panachure jaune du riz est endémique uniquement en Afrique, avec des pertes de production atteignant $100 \%$ sur les variétés hautement sensibles (Bouet et al., 2012; Issaka et al., 2012a ; Oludare et al., 2016).

Pour faire face à la maladie plusieurs gènes de résistance du riz au RYMV ont été identifiés au cours des dernières années (Sorho et al., 2005 ; Amancho et al., 2009). Deux types de résistance naturelle vis-à-vis du virus ont été répertoriés, suite à l'évaluation de nombreuses variétés de riz cultivées et sauvages (Kam et al., 2017). Il s'agit de la résistance dite élevée monogénique et de la résistance partielle polygénique. La résistance élevée, observée chez les variétés Giganté et Bekarossaka (O. sativa) (Albar et al., 2003 ; Rakotomalala et al., 2008) et chez quelques accessions de Tropical Oryza glaberrima (TOG) dont les variétés Tog5681, Tog5672, Tog5674, Tog5691 et Tog5307 (Thiémélé et al., 2010 ; Pidon et al., 2017), est due à trois (3) gènes de résistance, les gènes RYMVI, $R Y M V 2$ et $R Y M V 3$. Elle se manifeste, chez les deux espèces de riz, par une absence de symptômes de panachure jaune et/ou une faible teneur en virus; c'est une résistance récessive et stable qui est exploitable en sélection (Albar et al., 2003). Le gène RYMVI a été le mieux étudié et, est responsable de la haute résistance des variétés Giganté, Bekarossaka, Tog5681, Tog5672 et Tog5674 (Albar et al., 2003). Ce gène présente un allèle dominant de sensibilité RYMVI-1 chez la variété IR1529, sensible au Niger et 4 allèles récessifs de résistance rymvl-2 (Giganté et Bekarossaka), rymv1-3, rymv1-4 et rymv1-5 dans les accessions TOGs (Albar et al., 2006 ; Thiémélé et al., 2010). Plusieurs lignées de riz transgéniques ont aussi été développées, par introgression d'une partie ou de la séquence entière des gènes codant pour la polymérase virale ou la protéine de capside (Sorho et al., 2005 ; Kouassi et al., 2006).

Des méthodes de lutte basées sur l'utilisation en sélection des gènes de résistance au RYMV ont été développées et le déploiement des variétés résistantes dans les 
écologies rizicoles est préconisé (Sorho et al., 2005). Des résultats intéressants sur la diversité du virus et son épidémiologie dans plusieurs zones rizicoles sont aussi disponibles (Traoré et al., 2005; Traoré et al., 2009 ; Issaka et al., 2012a). Ainsi, la présence d'isolats contournants et/ou très agressifs a été rapportée dans plusieurs écologies rizicoles du continent (Traoré et al. 2006, Traoré et al., 2010 ; Issaka et al., 2012b ; Hebrard et al., 2018). Aussi, l'allèle de résistance rymv1-2 a, à l'aide de marqueurs moléculaires, a été transféré à un nombre limité de variétés améliorées de riz irrigué. Certaines de ces variétés ont été distribuées par le Centre du riz pour l'Afrique (AfricaRice) dans la plupart des pays de l'UEMOA, à travers le CORAF/WECARD et le PPAAO (Bouet et al., 2013). Le déploiement des variétés de riz dotées du gène RYMVI visant l'atténuation voire la résolution des problèmes de productivité du riz liés au virus est en cours dans plusieurs écologies rizicoles. En effet, des résultats prometteurs sont attendus et ils visent à s'affranchir des pertes énormes de la production du riz sur le continent.

La panachure jaune du riz a été décrite pour la première fois au Niger dans les années 1990 (Issaka, 2013). Elle y a fait son apparition suite à l'intensification de la riziculture. Aussi, une caractérisation épidémiologique de l'espace rizicole du Niger a rapporté la présence du virus dans tous les périmètres irrigués, avec des incidences et des pertes de récoltes avoisinant respectivement $90 \%$ et $71 \%$ (Issaka et al., 2012a). Il ressort que les principales variétés de riz cultivées au Niger et même les variétés résistantes développées par AfricaRice (WITA 8 et IR47686-15-1) sont sensibles au RYMV (Basso et al., 2010). De même, la présence d'isolats du virus capables de contourner la résistance des témoins de résistance (Giganté et Tog5681) a été rapportée dans cette écologie rizicole (Issaka et al., 2012b, Kam et al., 2018).

Cependant, il est relevé qu'il subsiste encore d'importants gaps d'informations sur la durabilité des génotypes améliorés (dotés de gènes de résistance) déployés dans les rizières du pays, étant donné que les données d'incidence et de pertes de productions de grains évoquées remontaient à 2008 ; plus d'une décennie, caractérisée par d'importants efforts de sensibilisations et/ou formations sur le contrôle de la maladie en champ (Issaka et al., Communication personnelle). Aussi, d'une part, la distribution spatiale des isolats virulents et/ou très agressifs dans les différentes zones rizicoles reste à compléter et d'autre part, le déploiement des gènes de résistance durables devrait s'effectuer en lien avec la distribution spatiale de la panachure jaune dans l'écologie rizicole du pays. Une réévaluation de l'ampleur de la maladie dans les rizières du Niger s'avère donc nécessaire voire un préalable à tout déploiement de gènes de résistance, pour estimer l'incidence et la distribution spatiale de la maladie et, évaluer les pertes de productions en champs ainsi que le niveau de résistance des variétés cultivées.

\section{MATERIEL ET METHODES}

\section{Evaluation de l'incidence de la panachure jaune du riz dans les sites prospectés}

Une prospection a été conduite dans 15 périmètres irrigués où le riz est cultivé. L'estimation de l'incidence de la panachure jaune du riz, faite selon la méthode de Issaka et al., (2012a), a consisté à visiter l'ensemble des périmètres rizicoles et à dénombrer ceux qui sont infectés, afin d'estimer leurs niveaux d'infestation. Les incidences ont été évaluées par comptage du nombre de pieds malades et sains sur trois lignes (espacées de $20 \mathrm{~cm}$ ) prises au hasard dans un champ de 0,25 ha. Pour estimer le pôle inférieur de l'incidence, le procédé a été le même, à la différence que le champ concerné est faiblement infesté.

Echantillonnage des sites rizicoles et inventaire des principales variétés cultivées

Tous les quinze périmètres rizicoles prospectés ont été échantillonnés. Des échantillons de feuilles de riz et de Graminées adventices présentant des symptômes typiques 
ou non de panachure jaune ont été prélevés dans et aux alentours des parcelles visitées. Lors du prélèvement, nous avions pris le soin d'envelopper les mains par des sachets plastiques et de retourner les sachets sans toucher aux feuilles avec les mains nues. Plus de 150 échantillons de feuilles de riz ou d'adventices présentant ou pas de symptômes de la panachure jaune du riz ont été collectés, conservés dans une glacière avec de la glace et acheminés au laboratoire où ils ont été analysés en condition de confinement pour respecter les règles de biosécurité.

Une enquête de recensement des principales variétés de riz cultivées a été conduite auprès des producteurs et des directions des coopératives des périmètres prospectés. Cette enquête associée à un constat de terrain sur l'effectivité de la culture desdites variétés a permis d'inventorier les variétés largement cultivées et d'avoir une idée de leur niveau de résistance vis-à-vis de la panachure jaune du riz.

\section{Evaluation des pertes de production de grains}

Lors de la prospection, des carrés de sondage d'un mètre carré $\left(1 \mathrm{~m}^{2}\right)$ de superficie ont été posés dans des plages apparemment saines et des plages malades des périmètres de Sébéri, Saga, Famalé, Toula, Kareygorou et Gaya Amont, afin de calculer les pertes de récoltes dues à la maladie en champ paysan. Les périmètres d'implantation des carrés de rendement ayant une large distribution spatiale ont été fortement infestés et identifiés endémiques lors de la prospection, avec de fortes incidences. La perte de production (PPd) est calculée à partir de l'Incidence (I) de la maladie et de la perte potentielle (Ppt) déduite des récoltes de grains des carrés de sondage, selon la formule appliquée par Issaka et al., (2012a), ci-après:

PPd $=(\operatorname{Ppt} \times$ I $) / 100$
Caractérisation biologique des échantillons collectés et identification des adventices porteuses du virus

Le matériel viral de caractérisation biologique du virus a été constitué de 152 échantillons dont 113 en feuilles de riz 39 adventices, suspectés contenir le virus et collectés sur l'ensemble des 15 sites prospectées. Ces échantillons ont été collectés durant les deux campagnes rizicoles de 2019. Chacun des échantillons de riz et d'adventices a été propagé sur 4 variétés de référence (Tableau 1) déjà décrites par Albar et al., (2003).

Les grains de chacune des variétés utilisées ont été pré-germés dans des boites de Pétri pendant $72 \mathrm{~h}$ avant d'être transplantés dans des pots (de 2 1) préalablement remplis de terre argileuse. La transplantation des plants a été faite, à raison de trois plants par pot et un pot par combinaison variété / isolat.

Les échantillons de feuilles ou isolats ont été broyés dans du tampon phosphate 0,1 $\mathrm{M} \mathrm{pH} \mathrm{7,2} \mathrm{(KH2Po4} \mathrm{0,1M} \mathrm{+} \mathrm{Na2HPo4} \mathrm{0,1M),}$ à raison de $1 \mathrm{~g}$ de feuilles pour $10 \mathrm{ml}$ de tampon d'inoculation. Du carborundum (600 mesh) a été additionné au broyat (à raison de $0,1 \mathrm{~g}$ pour $100 \mu \mathrm{l}$ de broyat), pour servir d'abrasif lors de l'inoculation (Onasanya et al., 2006). L'inoculation a lieu à 14 jours après transplantation (JAT). Elle a consisté à frotter chaque plant (excepté les témoins) avec l'inoculum. Les plants témoins ont été inoculés avec un inoculum constitué du tampon d'inoculation et de l'abrasif dans les mêmes concentrations. La maladie a été évaluée visuellement (sur la base des symptômes) à 21 jours après inoculation (JAI), à l'aide de l'échelle de sévérité $1-9$ où 1 représente l'absence de symptômes et 9 la mort des feuilles.

Les feuilles d'adventices du riz collectées au niveau des bordures et à l'intérieur des parcelles de riz ont été analysées, afin d'identifier lesdites adventices, à l'aide du Lexique des noms vernaculaires des plantes du Niger et du Weeds of Rice in West Africa. 
Tableau 1 : Caractéristiques des variétés utilisées dans la caractérisation biologique des échantillons de feuilles collectés en champ

\begin{tabular}{ccccc}
\hline Code & Génotype / Variété & Espèce / S/Espèce & Allèle de résistance & Références* \\
\hline V1 & Giganté & O. sativa (indica) & rymv1-2 & HR \\
V2 & Tog 5681 & O. glaberrima & rymv1-3 & HR \\
V3 & IR 64 & O. sativa (indica) & rymv1-1 & HS \\
V4 & IR 1529-680-3-1 & O. sativa (indica) & rymv1-1 & HS \\
\hline
\end{tabular}

(Source : Albar et al, 2006)

\section{RESULTATS \\ Incidence et distribution spatiale de la maladie}

La panachure jaune du riz a été observée dans presque tous les sites rizicoles prospectés, aussi bien sur le riz cultivé que chez les Graminées adventices, en saison sèche comme en saison humide (Tableau 2). La plus forte incidence de la maladie a été de $70 \%$, en saison humide et $30 \%$, en campagne sèche. L'incidence de la maladie au champ a varié entre 5 et $30 \%$ en saison sèche, et en saison humide, elle a oxillé entre 5 et $70 \%$, avec des niveaux d'infestation variables d'un périmètre à l'autre (Tableau 3). Les périmètres de Sébéri, Saga, Kareygorou, Famalé, Gaya et Toula ont été fortement infestés, avec des incidences allant jusqu'à $45-70 \%$ en saison humide. Par contre, dans les sites de Say1, Lata et Lossa, les niveaux d'infestation des rizières n'ont guère excédé $10 \%$ pendant la même saison. Toutefois, en saison sèche, ces périmètres ont été indemnes de panachure jaune.

La Figure 1 donne la distribution spatiale de la panachure jaune du riz dans les 15 périmètres prospectés. Elle montre que la maladie est largement distribuée dans l'écologie rizicole du pays, avec des incidences variables indépendantes de la position géographique du site. A titre illustratif, les sites de Daïbéri et Toula sont très proches l'un de l'autre, avec des incidences respectives de $25 \%$ et $70 \%$.

\section{Inventaire des variétés cultivées et pertes de récoltes enregistrées}

Les enquêtes auprès des paysans et des directions des coopératives rizicoles des sites visités ont révélé que les périmètres rizicoles ont quasiment tous deux campagnes, dont une en saison humide (juin-novembre) et l'autre en saison sèche (décembre-avril). Toutefois, le calendrier cultural est légèrement différent d'un périmètre à l'autre.

Deux variétés de riz sont principalement cultivées (Tableau 4) dans l'espace rizicole du Niger. Il s'agit des variétés IR1529-680-3-1 et Kogoni 91-1. La IR1529-680-3-1 est exclusivement cultivée en saison sèche dans tous les sites rizicoles irrigués. Quant à la Kogoni 91-1, elle est cultivée essentiellement en saison humide dans tous périmètres de l'étude. Deux autres variétés, Maï-aléwa et Bassirou-Mo, sont respectivement cultivées à côté des variétés dominantes dans trois (20\%) et deux $(13,33 \%)$ des sites rizicoles prospectés (Tableau 4), aussi bien en saison sèche qu'en campagne humide.

Les résultats des carrés de rendement placés dans les périmètres fortement infestés (Tableau 5) ont montré des pertes moyennes de récoltes de plus de $63 \%$ et $18 \%$, respectivement en saison humide et en saison sèche. En effet, les pertes de récoltes observées varient entre $4,59-18,14 \%$ en saison sèche et, $27,17-63,26 \%$ en saison humide. Par ailleurs, les pertes ont été variables selon les sites concernés. Les plus grandes pertes de récoltes enregistrées en saison sèche l'ont été à Toula (18.14\%) et les 
plus faibles pertes observées à Saga (4.59\%). En saison humide, la maladie s'est montrée encore plus dommageable à Toula (63.26\%) tandis que les plus faibles pertes de récoltes ont été enregistrées à Gaya (27.17\%). Des pertes intermédiaires et variables de récoltes ont été obtenues au niveau des quatre autres sites rizicoles (Tableau 5).

\section{Résultats biologiques et caractéristiques des isolats testés}

La présence de la panachure du riz dans l'écologie rizicole du Niger a été confirmée par les résultats biologiques, à travers les profils de réaction des isolats vis-àvis des quatre variétés testées (Tableaux 3 et 6). Il y ressort qu'au moins un échantillon provenant de chacun des 15 sites prospectés a pu produire des symptômes typiques de la maladie (Figure 2) sur les deux variétés hypersensibles (IR1529-680-3-1 et IR64); confirmant ainsi la présence de cette dernière dans les rizières du Niger.

Soixante-trois (63) isolats du RYMV ont été inventoriés, aussi bien en saison sèche qu'en saison humide (Tableau 6). Il s'agit de
43 isolats sur le riz cultivé et 20 sur les adventices Au nombre de ces isolats figurent les isolats sauvages qui n'ont infecté que les variétés sensibles (IR64 et IR1529-680-3-1) et les isolats de contournement de la résistance ou Resistance Breaking isolates (RB), virulents sur les variétés résistantes (Giganté et Tog5681). Ainsi, 6,34\% des isolats RB $(\mathrm{Ng} 25, \mathrm{Ng} 47, \mathrm{Ng} 57$ et $\mathrm{Ng} 63)$ ont été capables de contourner la résistance de Giganté (RBGiganté) en y produisant des symptômes de panachure jaune et 14,28\% d'entre eux ( $\mathrm{Ng} 16$, $\mathrm{Ng} 22, \mathrm{Ng} 24, \mathrm{Ng} 32, \mathrm{Ng} 41, \mathrm{Ng} 46, \mathrm{Ng} 56, \mathrm{Ng} 60$ et $\mathrm{Ng62}$ ) ont été virulents sur l'allèle de résistance rymv1-3 de Tog5681 (RBTog5681), sur la base des symptômes observés.

Les espèces d'adventices identifiées appartiennent toutes à la famille des Poacées. Il s'agit des espèces Eleusine indica, Echinocloa colona et Learsia hexandra (Tableau 3) présentes comme hôtes réservoirs du RYMV ; étant donné que leurs échantillons de feuilles ont produit des symptômes typiques de panachure jaune lors des tests biologiques.

Tableau 2: Caractéristiques des quinze (15) périmètres rizicoles prospectés et répartition des échantillons collectés.

\begin{tabular}{|c|c|c|c|c|c|c|c|c|}
\hline \multirow[b]{2}{*}{$\mathbf{S} / \mathbf{N}$} & \multirow[b]{2}{*}{ Périmètre } & \multirow[b]{2}{*}{$\begin{array}{l}\text { Campagn } \\
\text { e rizicole }\end{array}$} & \multirow[b]{2}{*}{$\begin{array}{l}\text { Superficie } \\
\text { (ha) }\end{array}$} & \multirow[b]{2}{*}{$\begin{array}{c}\text { Coordonnées } \\
\text { GPS }\end{array}$} & \multirow[b]{2}{*}{ Ecologie } & \multirow{2}{*}{$\begin{array}{c}\text { Nombre } \\
\text { d'échantillon } \\
\text { s collectés }\end{array}$} & \multicolumn{2}{|c|}{ Origine } \\
\hline & & & & & & & $\begin{array}{l}\text { Riz } \\
\text { cultivé }\end{array}$ & Adventice \\
\hline \multirow{2}{*}{1} & \multirow{2}{*}{ Saga } & \multirow{2}{*}{ SS 2019} & 323,75 & \multirow{2}{*}{$\begin{array}{c}\mathrm{N} 13^{\circ} 27.104^{\prime} ; \\
\text { E } 2^{\circ} 8.979^{\prime}\end{array}$} & Irriguée & 3 & 2 & 1 \\
\hline & & & 233,57 & & Irriguée & 11 & 8 & 3 \\
\hline \multirow{2}{*}{2} & \multirow{2}{*}{ Koutoukalé } & \multirow{2}{*}{ SS 2019} & 321,57 & \multirow{2}{*}{$\begin{array}{l}\text { N } 13^{\circ} 41.410^{\prime} ; \\
\text { E } 1^{\circ} 43.611^{\prime}\end{array}$} & Irriguée & 3 & 3 & 0 \\
\hline & & & 298,6 & & Irriguée & 6 & 5 & 1 \\
\hline \multirow{2}{*}{3} & \multirow{2}{*}{ Diomona } & \multirow{2}{*}{ SS 2019} & 391,25 & \multirow{2}{*}{$\begin{array}{l}\mathrm{N} 14^{\circ} 27.193^{\prime} ; \\
\text { E } 1^{\circ} 6.134^{\prime}\end{array}$} & Irriguée & 3 & 2 & 1 \\
\hline & & & 391,69 & & Irriguée & 6 & 4 & 2 \\
\hline \multirow{2}{*}{4} & \multirow{2}{*}{ Bonféba } & \multirow{2}{*}{ SS 2019} & 307 & \multirow{2}{*}{$\begin{array}{l}\mathrm{N} 14^{\circ} 22.286^{\prime} ; \\
\mathrm{E} 1^{\circ} 12.318^{\prime}\end{array}$} & Irriguée & 3 & 3 & 0 \\
\hline & & & 285 & & Irriguée & 5 & 4 & 1 \\
\hline
\end{tabular}




\begin{tabular}{|c|c|c|c|c|c|c|c|c|}
\hline \multirow{2}{*}{5} & \multirow{2}{*}{ Sébéri } & \multirow{2}{*}{ SS 2019} & 206,4 & \multirow{2}{*}{$\begin{array}{l}\text { N } 13^{\circ} 18.032^{\prime} \\
\text { E } 2^{\circ} 20.193^{\prime}\end{array}$} & Irriguée & 4 & 3 & 1 \\
\hline & & & 290,03 & & Irriguée & 10 & 7 & 3 \\
\hline \multirow{2}{*}{6} & \multirow{2}{*}{ Famalé } & \multirow{2}{*}{ SS 2019} & 598 & \multirow{2}{*}{$\begin{array}{c}\mathrm{N} 14^{\circ} 39.129^{\prime} \mathrm{E} \\
0^{\circ} 57.552^{\prime}\end{array}$} & Irriguée & 3 & 3 & 2 \\
\hline & & & 600 & & Irriguée & 12 & 10 & 2 \\
\hline \multirow{2}{*}{7} & \multirow{2}{*}{ Liboré } & \multirow{2}{*}{ SS 2019} & 220 & \multirow{2}{*}{$\begin{array}{l}\text { N } 13^{\circ} 23.192^{\prime} \\
\text { E } 2^{\circ} 12.029^{\prime}\end{array}$} & Irriguée & 3 & 3 & 0 \\
\hline & & & 240 & & Irriguée & 5 & 3 & 2 \\
\hline \multirow{2}{*}{8} & \multirow{2}{*}{ Say 1} & \multirow{2}{*}{ SS 2019} & & \multirow{2}{*}{$\begin{array}{l}\mathrm{N} 13^{\circ} 6.626^{\prime} ; \mathrm{E} \\
2^{\circ} 21.177^{\prime}\end{array}$} & Irriguée & 0 & 0 & 0 \\
\hline & & & 184,08 & & Irriguée & 2 & 2 & 0 \\
\hline \multirow{2}{*}{9} & \multirow{2}{*}{$\begin{array}{l}\text { Gaya } \\
\text { amont }\end{array}$} & \multirow{2}{*}{ SS 2019} & & \multirow{2}{*}{$\begin{array}{l}\text { N } 11^{\circ} 53.468^{\prime} \\
\text { E } 13^{\circ} 29.155^{\prime}\end{array}$} & Irriguée & 4 & 2 & 2 \\
\hline & & & 294 & & Irriguée & 10 & 8 & 2 \\
\hline \multirow{2}{*}{10} & \multirow{2}{*}{$\begin{array}{l}\text { Karey } \\
\text { Gorou }\end{array}$} & \multirow{2}{*}{ SS 2019} & 134,74 & \multirow{2}{*}{$\begin{array}{l}\text { N } 13^{\circ} 34.176^{\prime} \\
\text { E } 1^{\circ} 59.036^{\prime}\end{array}$} & Irriguée & 3 & 3 & 2 \\
\hline & & & 131,83 & & Irriguée & 10 & 6 & 4 \\
\hline \multirow{2}{*}{11} & \multirow{2}{*}{ Lata } & \multirow{2}{*}{ SS 2019} & 147,2 & \multirow{2}{*}{ 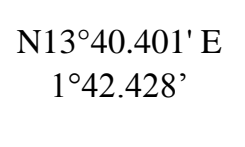 } & Irriguée & 0 & 0 & 0 \\
\hline & & & 141,3 & & Irriguée & 2 & 2 & 0 \\
\hline \multirow{2}{*}{12} & \multirow{2}{*}{ Daibéri } & \multirow{2}{*}{ SS 2019} & 296 & \multirow{2}{*}{ 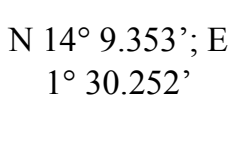 } & Irriguée & 3 & 2 & 1 \\
\hline & & & 296 & & Irriguée & 5 & 3 & 2 \\
\hline \multirow{2}{*}{13} & $\mathrm{Josca}$ & SS 2019 & 108 & $\mathrm{~N} 13^{\circ} 55.169^{\prime} \mathrm{E}$ & Irriguée & 0 & 0 & 0 \\
\hline & Lussa & (3) & 145,93 & $1^{\circ} 34.172$ & Irriguée & 2 & 2 & 0 \\
\hline 14 & Namari & SS 2019 & 660 & N 14² 21.597’; & Irriguée & 4 & 3 & 1 \\
\hline $1+1$ & Goungou & (ל) & 660 & $\mathrm{E} 1^{\circ} 14.396^{\prime}$ & Irriguée & 5 & 3 & 2 \\
\hline 15 & Toula & SS 2019 & 243,74 & N 14 11.490'; & Irriguée & 6 & 5 & 1 \\
\hline & 18um & 年 & 243,74 & E $1^{\circ} 27.602^{\prime}$ & Irriguée & 15 & 12 & 3 \\
\hline & & & & & Total & 152 & 113 & 39 \\
\hline
\end{tabular}

SS : saison sèche ; $\mathrm{SH}$ : saison humide. 
Tableau 3 : Incidence de la panachure jaune du riz et principales espèces d'adventices issues des tests biologiques préliminaires.

\begin{tabular}{|c|c|c|c|c|c|c|}
\hline \multirow{2}{*}{$\mathbf{S} / \mathbf{N}$} & \multirow{2}{*}{ Périmètre } & \multirow{2}{*}{$\begin{array}{l}\text { Campagne } \\
\text { rizicole }\end{array}$} & \multirow{2}{*}{$\begin{array}{l}\text { Incidence } \\
(\%)\end{array}$} & \multicolumn{2}{|c|}{ Origine / isolats } & \multirow{2}{*}{ Espèce d'adventices } \\
\hline & & & & Riz cultivé & Adventices & \\
\hline \multirow{2}{*}{1} & \multirow{2}{*}{ Saga } & SS 2019 & 15 & 1 & 0 & - \\
\hline & & SH 2019 & 50 & 3 & 1 & Eleusine indica \\
\hline \multirow{2}{*}{2} & \multirow{2}{*}{ Koutoukalé } & SS 2019 & 5 & 1 & 0 & - \\
\hline & & SH 2019 & 30 & 2 & 1 & Echinochloa colona \\
\hline \multirow{2}{*}{3} & \multirow{2}{*}{ Diomona } & SS 2019 & 5.00 & 1 & 0 & - \\
\hline & & SH 2019 & 30 & 2 & 1 & Learsia hexandra \\
\hline \multirow{2}{*}{4} & \multirow{2}{*}{ Bonféba } & SS 2019 & 10 & 1 & 0 & - \\
\hline & & SH 2019 & 40 & 2 & 1 & Learsia hexandra \\
\hline \multirow[b]{2}{*}{5} & \multirow[b]{2}{*}{ Sébéri } & SS 2019 & 20 & 2 & 1 & Eleusine indica \\
\hline & & SH 2019 & 45 & 3 & 2 & $\begin{array}{l}\text { Echinochloa colona, Lersia } \\
\text { hexandra }\end{array}$ \\
\hline \multirow[b]{2}{*}{6} & \multirow[b]{2}{*}{ Famalé } & SS 2019 & 25 & 1 & 0 & - \\
\hline & & SH 2019 & 55 & 5 & 2 & $\begin{array}{l}\text { Learsia hexandra, Eleusine } \\
\text { indica }\end{array}$ \\
\hline \multirow{2}{*}{7} & \multirow{2}{*}{ Liboré } & SS 2019 & 15 & 1 & 0 & - \\
\hline & & SH 2019 & 25 & 2 & 1 & Learsia hexandra \\
\hline \multirow{2}{*}{8} & \multirow{2}{*}{ Say 1} & SS 2019 & 0 & 0 & 0 & - \\
\hline & & SH 2019 & 10.00 & 1 & 0 & - \\
\hline \multirow{2}{*}{9} & \multirow{2}{*}{ Gaya amont } & SS 2019 & 25 & 1 & 1 & Eleusine indica \\
\hline & & SH 2019 & 45 & 2 & 1 & Learsia hexandra \\
\hline \multirow{2}{*}{10} & \multirow{2}{*}{ Karey Gorou } & SS 2019 & 25 & 0 & 2 & $\begin{array}{c}\text { Echinochloa colona, Eleusine } \\
\text { indica }\end{array}$ \\
\hline & & SH 2019 & 45 & 2 & 1 & Echinochloa colona \\
\hline \multirow{2}{*}{11} & \multirow{2}{*}{ Lata } & SS 2019 & 0 & 0 & 0 & - \\
\hline & & SH 2019 & 10.00 & 1 & 0 & - \\
\hline \multirow{2}{*}{12} & Daibéri & SS2019 & 15.00 & 1 & 0 & - \\
\hline & Daivent & SH 2019 & 25.00 & 1 & 1 & Eleusine indica \\
\hline 13 & $\mathrm{I} / \mathrm{scc}$ & SS 2019 & 0 & 0 & 0 & - \\
\hline 15 & LOssa & SH 2019 & 5.00 & 1 & 0 & - \\
\hline 14 & Namari & SS 2019 & 15.00 & 1 & 0 & \\
\hline 14 & Goungou & SH 2019 & 35.00 & 1 & 1 & Eleusine indica \\
\hline & & SS 2019 & 30.00 & 1 & 1 & Eleusine indica \\
\hline 15 & Toula & SH 2019 & 70.00 & 3 & 2 & $\begin{array}{l}\text { Eleusine indica, Echinochloa } \\
\text { colona }\end{array}$ \\
\hline & & & Total & 43 & 20 & \\
\hline
\end{tabular}

SS : saison sèche ; SH : saison humide ; - : aucune adventice identifiée. 


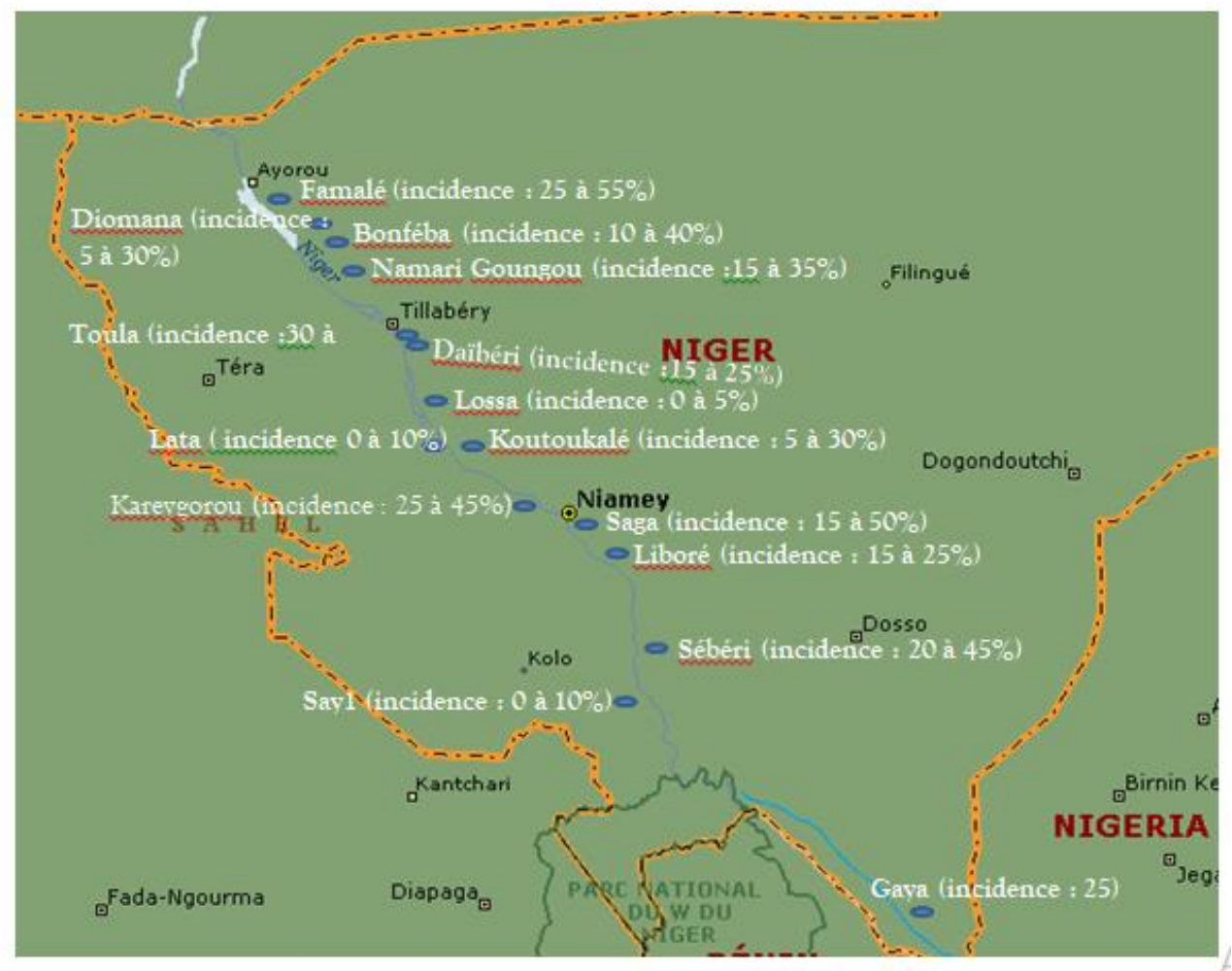

Figure 1 : Distibution spatiale des sites rizicoles prospectés en 2019 (emplacement des sites et incidence de la maladie en $\%$ ).

Tableau 4 : Répartition des variétés de riz emblavées dans les périmètres rizicoles prospectés.

\begin{tabular}{|c|c|c|c|c|c|c|}
\hline \multirow{2}{*}{$\mathbf{S} / \mathbf{N}$} & \multirow{2}{*}{ Périmètre } & \multirow{2}{*}{$\begin{array}{l}\text { Campagne } \\
\text { rizicole }\end{array}$} & \multicolumn{4}{|c|}{ Variétés emblavées* } \\
\hline & & & IR $1529-680-3^{\mathrm{HS}}$ & Kogoni 90-1 ${ }^{\mathrm{MR}}$ & Maï aléwa ${ }^{\mathrm{VSS}}$ & BassirouMo $^{\mathrm{VSS}}$ \\
\hline \multirow{2}{*}{1} & \multirow{2}{*}{ Saga } & SS 2019 & $\mathrm{x}$ & & & \\
\hline & & SH 2019 & & $\mathrm{x}$ & & \\
\hline \multirow{2}{*}{2} & \multirow{2}{*}{ Koutoukalé } & SS 2019 & $\mathrm{x}$ & & & \\
\hline & & SH 2019 & & $\mathrm{x}$ & & \\
\hline \multirow{2}{*}{3} & \multirow{2}{*}{ Diomona } & SS 2019 & $\mathrm{x}$ & & & $\mathrm{x}$ \\
\hline & & SH 2019 & & $\mathrm{x}$ & & \\
\hline \multirow{2}{*}{4} & \multirow{2}{*}{ Bonféba } & SS 2019 & $\mathrm{x}$ & & & $\mathrm{x}$ \\
\hline & & SH 2019 & & $\mathrm{x}$ & & \\
\hline \multirow{2}{*}{5} & \multirow{2}{*}{ Sébéri } & SS 2019 & $x$ & & & \\
\hline & & SH 2019 & & $\mathrm{x}$ & & \\
\hline \multirow{2}{*}{6} & \multirow{2}{*}{ Famalé } & SS 2019 & $\mathrm{x}$ & & & \\
\hline & & SH 2019 & & $\mathrm{x}$ & & \\
\hline \multirow{2}{*}{7} & \multirow{2}{*}{ Liboré } & SS 2019 & $\mathrm{x}$ & & $\mathrm{x}$ & \\
\hline & & SH 2019 & & $\mathrm{x}$ & $\mathrm{x}$ & \\
\hline
\end{tabular}




\begin{tabular}{|c|c|c|c|c|c|c|}
\hline \multirow{2}{*}{8} & \multirow{2}{*}{ Say 1} & SS 2019 & \multirow[t]{2}{*}{$\mathrm{x}$} & \multicolumn{3}{|c|}{$\mathrm{x}$} \\
\hline & & SH 2019 & & $\mathrm{x}$ & $\mathrm{x}$ & \\
\hline \multirow{2}{*}{9} & \multirow{2}{*}{ Gaya amont } & SS 2019 & $\mathrm{x}$ & & $\mathrm{x}$ & \\
\hline & & SH 2019 & & $\mathrm{x}$ & $\mathrm{x}$ & \\
\hline \multirow{2}{*}{10} & \multirow{2}{*}{ Karey Gorou } & SS 2019 & $\mathrm{x}$ & & & \\
\hline & & SH 2019 & & $\mathrm{x}$ & & \\
\hline \multirow{2}{*}{11} & \multirow{2}{*}{ Lata } & SS 2019 & $\mathrm{x}$ & & & \\
\hline & & SH 2019 & & $\mathrm{x}$ & & \\
\hline \multirow{2}{*}{12} & \multirow{2}{*}{ Daibéri } & SS 2019 & $\mathrm{x}$ & & & \\
\hline & & SH 2019 & & $\mathrm{x}$ & & \\
\hline \multirow{2}{*}{13} & \multirow{2}{*}{ Lossa } & SS 2019 & $\mathrm{x}$ & & & \\
\hline & & SH 2019 & & $\mathrm{x}$ & & \\
\hline \multirow{2}{*}{14} & Namari & SS 2019 & $\mathrm{x}$ & & & \\
\hline & Goungou & SH 2019 & & $\mathrm{x}$ & & \\
\hline \multirow{2}{*}{15} & \multirow{2}{*}{ Toula } & SS 2019 & $\mathrm{x}$ & & & \\
\hline & & SH 2019 & & $\mathrm{x}$ & & \\
\hline \multirow{2}{*}{\multicolumn{2}{|c|}{$\begin{array}{c}\text { Nombre de sites } \\
\text { emblavés }\end{array}$}} & SS 2019 & 15 & $\mathbf{0}$ & 3 & 2 \\
\hline & & SH 2019 & $\mathbf{0}$ & 100 & 3 & 2 \\
\hline \multirow{2}{*}{\multicolumn{2}{|c|}{ Fréquence }} & SS 2019 & $100 \%$ & $0 \%$ & $20 \%$ & $13.33 \%$ \\
\hline & & SH 2019 & $0 \%$ & $100 \%$ & $20 \%$ & $13.33 \%$ \\
\hline
\end{tabular}

*MR : variété moyennement résistante ; HS : variété hautement sensible et VVS : variété sensible issue de la sélection massale paysanne à partir de la IR1529; VVS* : variété sensible issue de la sélection massale paysanne à partir d'un génotype (non caractérisé) venu du Nigéria voisin ; x : culture de la variété correspondante pendant la saison indiquée.

Tableau 5 : Les pertes économiques dues au RYMV.

\begin{tabular}{|c|c|c|c|c|c|}
\hline $\mathbf{S} / \mathbf{N}$ & Périmètre & Campagne & Incidence $(\%)$ & $\begin{array}{l}\text { Pertes potentielles de } \\
\text { productions* }(\%)\end{array}$ & Pertes de récoltes (\%) \\
\hline \multirow{2}{*}{1} & \multirow{2}{*}{ Saga } & SS 2019 & 15 & 30,58 & 4,59 \\
\hline & & SH 2019 & 50 & 80,33 & 40,17 \\
\hline \multirow{2}{*}{2} & \multirow{2}{*}{ Sébéri } & SS 2019 & 20 & 30,25 & 6,05 \\
\hline & & SH 2019 & 45 & 60,75 & 27,34 \\
\hline \multirow{2}{*}{3} & \multirow{2}{*}{ Famalé } & SS 2019 & 25 & 40 & 10,00 \\
\hline & & SH 2019 & 55 & 80,9 & 44,50 \\
\hline \multirow{2}{*}{4} & \multirow{2}{*}{ Gaya amont } & SS 2019 & 25 & 35,86 & 8,97 \\
\hline & & SH 2019 & 45 & 60,38 & 27,17 \\
\hline \multirow{2}{*}{5} & \multirow{2}{*}{ Karey Gorou } & SS 2019 & 25 & 55 & 13,75 \\
\hline & & SH 2019 & 45 & 65,73 & 29,58 \\
\hline \multirow{2}{*}{6} & \multirow{2}{*}{ Toula } & SS 2019 & 30 & 60,45 & 18,14 \\
\hline & & SH 2019 & 70 & 90,37 & 63,26 \\
\hline
\end{tabular}

*Pertes de production déduites des carrés de rendement. 


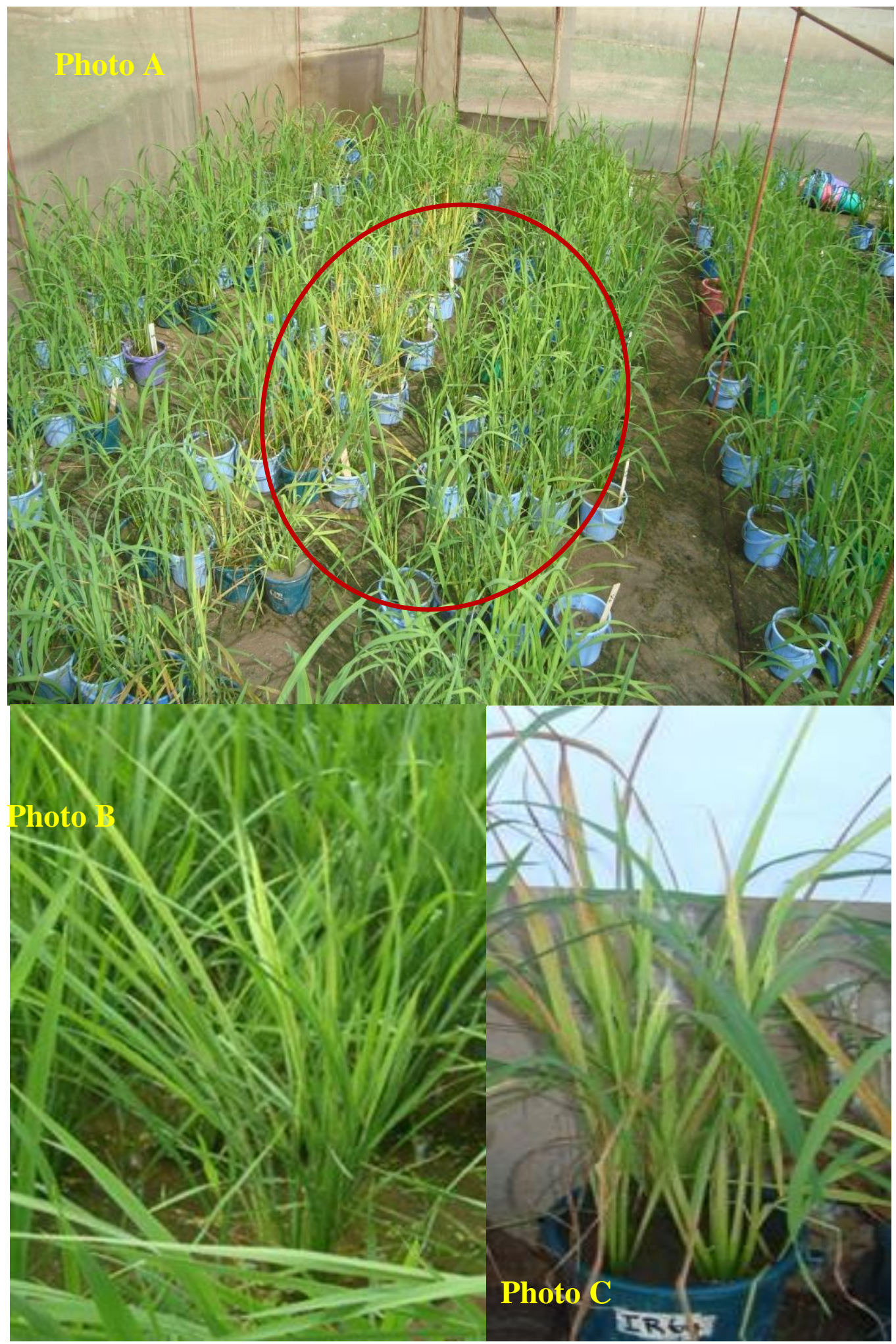

Figure 2 : Symptômes caractéristiques de panachure jaune du riz.

(Photo A : en serre à Kollo ; Photo B : en champ paysan à Liboré et Photo C : sur plant infecté en pot). 
Tableau 6 : Profil de réactions biologiques des isolats collectés.

\begin{tabular}{|c|c|c|c|c|c|c|c|}
\hline \multirow{2}{*}{$\mathbf{S} / \mathbf{N}$} & \multirow{2}{*}{ Périmètre } & \multirow{2}{*}{$\begin{array}{c}\text { Campagne } \\
\text { rizicole }\end{array}$} & \multirow{2}{*}{ Origine / isolats } & \multicolumn{4}{|c|}{ Variétés testées } \\
\hline & & & & IR1929 & IR64 & Giganté & Tog5681 \\
\hline \multirow{5}{*}{1} & \multirow{5}{*}{ Saga } & SS 2019 & Riz cultivé ( $\mathrm{Ng} 1)$ & + & + & - & - \\
\hline & & \multirow{4}{*}{ SH 2019} & Eleusine indica (Ng2) & + & + & - & - \\
\hline & & & Riz cultivé (Ng3) & + & + & - & - \\
\hline & & & Riz cultivé ( $\mathrm{Ng} 4)$ & + & + & - & - \\
\hline & & & Riz cultivé(Ng5) & + & + & - & - \\
\hline \multirow{4}{*}{2} & \multirow{4}{*}{ Koutoukalé } & SS 2019 & Riz cultivé (Ng6) & + & + & - & - \\
\hline & & & Echinochloa colona (Ng7) & + & + & - & - \\
\hline & & SH 2019 & Riz cultivé(Ng8) & + & + & - & - \\
\hline & & & Riz cultivé (Ng9) & + & + & - & - \\
\hline \multirow{4}{*}{3} & \multirow{4}{*}{ Diomona } & SS 2019 & Riz cultivé (Ng10) & + & + & - & - \\
\hline & & & Learsia hexandra (Ng11) & + & + & - & - \\
\hline & & SH 2019 & Riz cultivé(Ng12) & + & + & - & - \\
\hline & & & Riz cultivé (Ng13) & + & + & - & - \\
\hline \multirow{4}{*}{4} & \multirow{4}{*}{ Bonféba } & SS 2019 & Riz cultivé (Ng14) & + & + & - & - \\
\hline & & & Learsia hexandra (Ng15) & + & + & - & - \\
\hline & & SH 2019 & Riz cultivé (Ng16) & + & + & - & + \\
\hline & & & Riz cultivé (Ng17) & + & + & - & - \\
\hline \multirow{8}{*}{5} & \multirow{8}{*}{ Sébéri } & \multirow{4}{*}{ SS 2019} & Eleusine indica (Ng18) & + & + & - & - \\
\hline & & & Riz cultivé (Ng19) & + & + & - & - \\
\hline & & & Riz cultivé (Ng20) & + & + & - & + \\
\hline & & & Echinochloa colona (Ng21) & + & + & - & - \\
\hline & & \multirow{4}{*}{ SH 2019} & Lerasia hexandra (Ng22) & + & + & - & + \\
\hline & & & Riz cultivé (Ng23) & + & + & - & - \\
\hline & & & Riz cultivé (Ng24) & + & + & - & + \\
\hline & & & Riz cultivé (Ng25) & + & + & + & - \\
\hline \multirow{8}{*}{6} & \multirow{8}{*}{ Famalé } & SS 2019 & Riz cultivé (Ng26) & + & + & - & - \\
\hline & & \multirow{7}{*}{ SH 2019} & Learsia hexandra (Ng27) & + & + & - & + \\
\hline & & & Eleusine indica (Ng28) & + & + & - & - \\
\hline & & & Riz cultivé (Ng29) & + & + & - & - \\
\hline & & & Riz cultivé (Ng30) & + & + & - & + \\
\hline & & & Riz cultivé (Ng31) & + & + & - & - \\
\hline & & & Riz cultivé (Ng32) & + & + & - & + \\
\hline & & & Riz cultivé (Ng33) & + & + & - & - \\
\hline \multirow{4}{*}{7} & \multirow{4}{*}{ Liboré } & SS 2019 & Riz cultivé (Ng34) & + & + & - & - \\
\hline & & \multirow{3}{*}{ SH 2019} & Learsia hexandra (Ng35) & + & + & - & - \\
\hline & & & Riz cultivé (Ng36) & + & + & - & - \\
\hline & & & Riz cultivé (Ng37) & + & + & - & - \\
\hline 8 & Say 1 & SH 2019 & Riz cultivé (Ng38) & + & + & - & - \\
\hline 0 & & & Eleusine indica (Ng39) & + & + & - & - \\
\hline 9 & Gaya & SS 2019 & Riz cultivé (Ng40) & + & + & - & - \\
\hline
\end{tabular}


SH 2019

SS 2019

$10 \quad \begin{aligned} & \text { Karey } \\ & \text { Gorou }\end{aligned}$

SH 2019

11 Lata

SH 2019

SS 2019

12 Daibéri

SH 2019

13 Lossa

SH 2019

SS 2019

14

Namari

Goungou

SH 2019

SS 2019

15 Toula

SH 2019

Learsia hexandra $(\mathrm{Ng} 41)$

Riz cultivé $(\mathrm{Ng} 42)$

Riz cultivé ( $\mathrm{Ng} 43)$

Eleusine indica ( $\mathrm{Ng} 44)$

Echinochloa colona ( $\mathrm{Ng} 45)$

Echinochloa colona (Ng46)

Riz cultivé ( $\mathrm{Ng} 47)$

Riz cultivé ( $\mathrm{Ng} 48$ )

Riz cultivé ( $\mathrm{Ng} 49)$

Riz cultivé ( $\mathrm{Ng} 50)$

Eleusine indica (Ng51)

Riz cultivé (Ng52)

Riz cultivé ( $\mathrm{Ng} 53$ )

Riz cultivé ( $\mathrm{Ng} 54)$

Eleusine indica ( $\mathrm{Ng} 5)$

Riz cultivé ( $\mathrm{Ng} 56)$

Eleusine indica ( $\mathrm{Ng} 57)$

Riz cultivé (Ng58)

Eleusine indica (Ng59)

Echinochloa colona ( $\mathrm{Ng60)}$

Riz cultivé ( $\mathrm{Ng} 61)$

Riz cultivé ( $\mathrm{Ng62}$

Riz cultivé ( $\mathrm{Ng} 63$ )

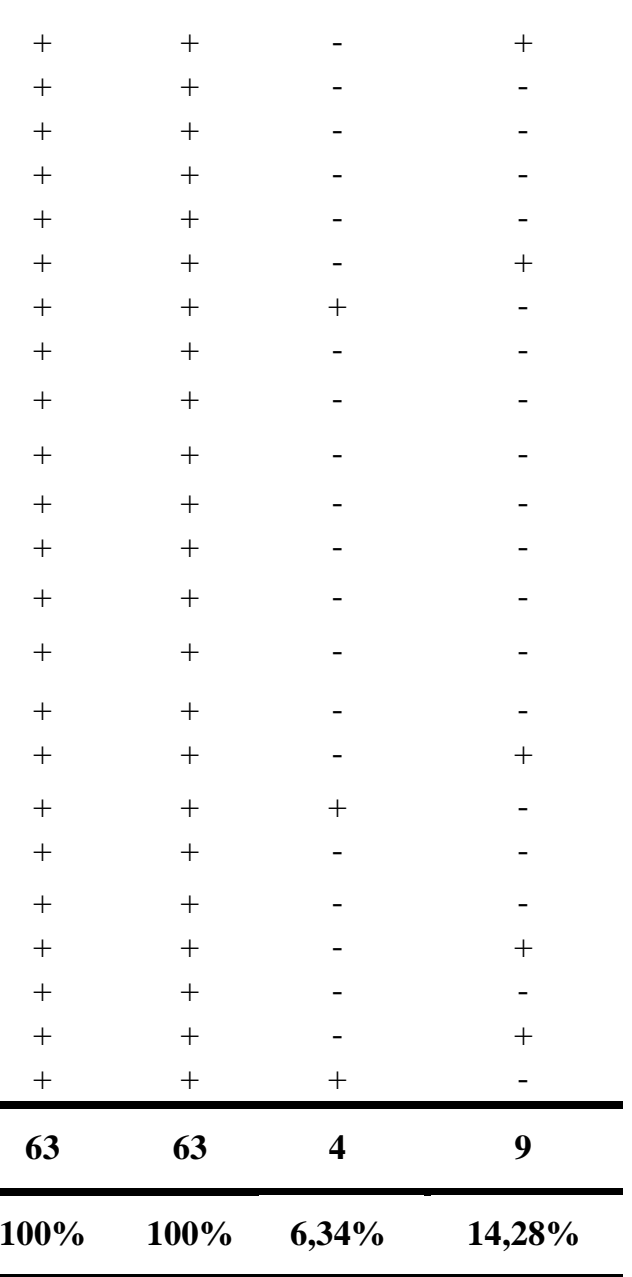

Fréquence des isolats

+ Positif sur la variété ; - Négatif sur la variété

\section{DISCUSSION}

Cette étude de réévaluation de la panachure jaune du riz au Niger a montré que la maladie est toujours présente dans l'écologie rizicole du pays. La distribution géographique des sites prospectés suggère que la maladie occupe toujours les principaux sites rizicoles et elle constitue une contrainte biotique majeure de la production rizicole. L'incidence de la maladie, variable d'un périmètre à un autre, est plus forte en saison humide où elle atteint $70 \%$ alors qu'en saison sèche, elle ne dépasse guère $30 \%$. Selon Issaka et al. (2012a), l'importance de la maladie en saison humide s'expliquerait à la fois par la grande pullulation des vecteurs à cette période suite à l'amélioration des conditions climatiques et alimentaires et par la présence massive des sources d'inoculum (hôtes réservoirs et autres). Par contre, en campagne sèche, la faible présence des insectes vecteurs et des hôtes réservoirs, due à la détérioration des conditions de l'habitat, limiterait considérablement la masse d'inoculum, son transfert et sa propagation au champ.

Les valeurs d'incidence enregistrées (maximum 70\%) diffèrent de celles de Issaka et al. (2012a), et Kouassi et al. (2005) qui ont rapporté des incidences maximales respectives de $90 \%$ et $100 \%$ dans l'écologie rizicole du Niger. $\mathrm{Ce}$ qui indiquerait une légère atténuation de la panachure jaune du riz au Niger. Cependant, le niveau d'infestation des rizières du pays par la maladie reste donc très préoccupant, étant donné que pratiquement 
seules deux variétés, dont l'une est très sensible (IR1529) et l'autre modérément résistante (Kogoni91-1), sont les plus cultivées dans l'espace rizicole. Ce qui constitue un risque d'explosion de la panachure jaune du riz, eu égard à la très forte étroitesse de la base génétique des variétés cultivées (Issaka et al., 2012a). En effet, l'avènement fortuit d'un variant de RYMV, virulent sur la Kogoni91-1, modérément résistante et largement cultivée en saison humide, pourrait produire une grande masse d'inoculum dans l'écologie rizicole irriguée, par le biais des insectes vecteurs qui pullulent abondamment pendant cette saison. De même, l'apparition d'une poche d'infection en saison sèche, quelque part dans une rizière environnante, pourrait conduire à l'envahissement de la variété IR1529-680-3-1 cultivée sur un grand nombre de rizières, via les outils de travail, les pratiques culturales, et autres voies de contamination (insectes, eau d'irrigation, vent, etc.). En effet, les pratiques culturales et les autres voies de contamination favoriseraient fortement la dispersion du virus dans l'ensemble des rizières en présence exclusive de la variété IR1529-680-3-1, hypersensible et meilleure source de multiplication du RYMV.

L'effet des contaminations via les hôtes réservoirs risquerait aussi d'être accentué par la présence des trois espèces d'adventices identifiées par la présente étude dans l'espace rizicole du Niger. L'espèce Echinochloa colona y a été rapportée pour la première fois comme hôte réservoir du RYMV et les espèces Eleusine indica et Learsia hexandra déjà signalée dans ledit espace Issaka et al. (2012a). Des scénarii de propagation de la maladie en champ, via les vecteurs, les hôtes réservoirs et les différents facteurs de dissémination ont été largement discutés par divers auteurs (Sara et al., 2004; Traoré et al., 2006; Traoré et al., 2009; Bouet et al., 2012).

Les pertes moyennes de production enregistrées en champ paysan sont variables selon les sites et la saison. Elles sont plus importantes en saison humide où elles oscillent entre $27,17 \%$ et $63,26 \%$. Ces pertes confirment la nocuité de la maladie et sont légèrement inférieures à celles de $71 \%$ rapportées par Issaka et al. (2012a). Ce qui indique que la panachure jaune reste toujours endémique et très préoccupante dans l'écologie rizicole du Niger. Ces pertes enregistrées en champ paysan, par contre sont différentes de celles de $56 \%$ précédemment rapportées (Kouassi et al., 2005). Aussi, des pertes de production atteignant $100 \%$ ont-elles auparavant été rapportées dans les périmètres de Liboré, saga et Saadia au Niger (Issaka, 2013). Récemment encore Oludaré et al., (2016) ont enregistré des pertes de production de $100 \%$ à Saga, Lossa et Toula toujours au Niger et, des fois, l'abandon total des champs y a été observé (Issaka, Communication personnelle). Ces importantes pertes de productions se justifieraient d'une part par l'étroitesse de la base génétique des variétés cultivées et la forte incidence de la maladie et, d'autre part, par la présence des conditions et facteurs propices à la dispersion de l'inoculum (présence des vecteurs et d'hôtes réservoirs, augmentation de la quantité d'inoculum et sa diffusion accélérée dans l'environnement) telles que dissertées par Issaka et al. (2012a). Des pertes de productions variant entre 17 et $100 \%$ ont aussi été enregistrées dans diverses écologies rizicoles de l'Afrique (Soko et al., 2015; Oludaré et al., 2016 ; Kam et al., 2017).

Les résultats biologiques enregistrés ont confirmé à leur tour la large distribution spatiale du virus dans les rizières du Niger, à travers la présence des isolats RYMV dans chaque site. Deux pathogroupes majeurs du virus y ont été identifiés. Il s'agit des isolats sauvages qui n'agressent que les variétés sensibles et des isolats RB (RB-Gignaté et RB-Tog5681) qui contournent la haute résistance de Giganté et Tog5681. Les isolats RB-Giganté représentent 14,76\% des isolats, et ils ne sont présents qu'à Sébéri, Kareygorou et Toula. Les RB-Tog5681, eux, sont inféodés aux sites de Toula, Namari Goungou, Kareygorou, Gaya, Sébéri, Famalé et Bonféba ; ils représentent $14,28 \%$ des isolats obtenus. Ce qui confirme la présence endémique des isolats $\mathrm{RB}$ au Niger. La présence des isolats RB dans l'espace rizicole 
du pays a été signalée, il y a plus d'une décennie, par Issaka et al., (2012b). Ces derniers avaient observé, à la fois sur la base des symptômes visuels et des mutations de contournement, des isolats RB-Giganté, RBTog5681 et RB-Tog5672 (résultats non publiés), avec une certaine proportion d'isolats double RB (RB-Giganté/Tog5681). Par ailleurs, la présence des isolats contournant la résistance des allèles rymv1-2, rymv1-3, rymv1-4 et rymv1-5 a été rapportée dans d'autres écologies rizicoles d'Afrique de l'Ouest (Burkina Faso, Mali, Côte d'ivoire, Niger) par Traoré et al. (2010) et Kam et al. (2018). Ces derniers avaient même signalé la présence d'un isolat double contournant, RBGiganté/Tog5681, au Mali.

La présente étude a montré la prévalence des isolats RB au Niger mais sans double contournant. Ce qui suggère que le pyramidage des gènes comme moyen efficace de lutte contre le RYMV décrit par Sorho et al. (2005) n'est pas nécessaire. Un déploiement d'allèle unique de résistance, efficient en fonction des écologies, pourrait être suffisant. Toutefois, une bonne connaissance de la carte de distribution des isolats RB associée à de nouvelles investigations sur ces types d'isolats, à travers tout l'espace rizicole du pays, constitue un préalable; la diffusion desdits allèles devant se faire au cas par cas. Les isolats RB, identifiés ici, serviraient à caractériser les collections variétales nationales des pays endémiques, afin d'y identifier des sources de résistance et de développer des variétés capables de contrer la propagation du RYMV dans l'espace rizicole africain, seul concerné par l'épidémie (Thiémélé et al., 2010; Bouet et al., 2012). Ce qui contribuera, à terme, à la durabilité de la résistance des génotypes en champ préconisée par Sorho et al. (2005).

\section{Conclusion}

La panachure jaune du riz occupe toujours les principaux sites rizicoles du Niger. L'incidence et les pertes de production enregistrées oxillent respectivement autour de $70 \%$ et $63 \%$. Elles ont été variables selon les saisons et les périmètres. Cependant, un risque d'explosion de la maladie pourrait être envisagé dans cette écologie rizicole irriguée, eu égard à la monoculture exclusive des variétés IR1529-680-3-1 et Kogoni91-1 et la présence quasi-permanente des adventices hôtes réservoirs et autres voies de dispersion de l'inoculum du RYMV. Aussi, la prévalence des isolats RB dans cette écologie pourraitelle constituer un handicap majeur au déploiement des gènes et/ou à la durabilité de la résistance en champ paysan. Toutefois, la connaissance de la distribution spatiale des isolats RB et leur l'utilisation dans les programmes de sélection variétale seraient une approche très intéressante dans le contrôle de la maladie au niveau de la zone d'étude voire sur tout le continent.

\section{CONFLITS D'INTERETS}

Les auteurs déclarent ne pas avoir de conflits d'intérêts.

\section{CONTRIBUTIONS DES AUTEURS}

SI et MAMS ont implémenté le protocole, conduit la prospection et l'échantillonnage, mis en place les essais biologiques, collecté, analysé et interprété les données, écrit et corrigé le manuscrit. $\mathrm{AB}$ a contribué à analyser et interpréter les données, et à écrire puis corriger le manuscrit.

\section{REMERCIEMENTS}

$\mathrm{Au}$ terme de l'étude, les auteurs remercient les reviewers pour avoir accepté de corriger le manuscrit. Ils remercient aussi M. MAHAMADOU Bizo, Technicien du développement agricole à l'INRAN/CERRA de Kollo, pour son appui technique dans la conduite des essais de caractérisation biologique des isolats collectés.

\section{REFERENCES}

Albar L, Ndjiondjop MN, Esshak Z, Berger A, Pinel A, Jones M, Fargette D, Ghesquière A. 2003. Fine genetic mapping of a gene required for Rice yellow mottle virus cell-to-cell movement. Theor Appl. Genet., 107(2): 371-378.

DOI: 
http://dx.doi.org/10.1007/s00122-0031258-4.

Albar L, Bangratz-Reyser M, Hebrard E, Ndjiondjop MN, Jones $\mathrm{M}$, Ghesquière A. 2006. Mutations in the eIF(iso) $4 \mathrm{G}$ translation initiation factor confer high resistance of rice to Rice yellow mottle virus. Plant J., 47(3): 417-426. DOI: http://dx.doi.org/10.1111/j.1365313X.2006.02792.x

Amancho NA, Kouassi NK, Diallo HA, Bouet A, Sangaré A, Kouadio JY. 2009. The Report of Highly Resistance-breaking isolates of Rice Yellow Mottle Virus in Côte d'Ivoire. The African Journal of plant Science and Biotechnology, 3(1): 44-50.

Basso A, Issaka S, Haougui A, Séré Y, Halidou A. 2010. Caractérisation biologique d'isolats du virus de la panachure jaune du riz au Niger. Annales de l'Université Abdou Moumouni, Tome XI-A : 112-119.

Bouet A, Amancho AN, Sanogo S, Camara M. 2012. Effet de la fertilisation azotée et phosphorée sur le développement de la Panachure jaune en riziculture aquatique en Côte d'Ivoire. Int. J. Biol. Chem. Sci., 6(6): 4071-4079. DOI: http://dx.doi.org/10.4314/ijbcs.v6i6.17.

Bouet A, Amancho AN, Kouassi N, Anguete K. 2013. Comportement de nouvelles lignées isogéniques de riz irrigué dotées du gène de résistance (rymv1) au RYMV en Afrique de l'ouest: situation en Côte d'Ivoire. Int. J. Biol. Chem. Sci., 7(3): 1221-1223.

DOI: https://doi.org/10.4314/ijbcs.v7i3.28.

Fargette D, Konaté G, Fauquet C, Muller E, Petershmitt M, Thresh J. 2006. Molecular

ecology and emergence of tropical plant viruses. Annu. Rev. Phytopath., 44(1): 235-260.

DOI:

https://doi.org/10.1146/annurev.phyto.44 .120705.104644.

Hebrard E, Pinel-Galzi A, Oludaré A, Poulicard N, Aribi J, Fabre S, Issaka S, Mariac C, Dereeper A, Albar L, Silue D, Fargette D. 2018. Identification of a
Hypervirulent Pathotype of Rice yellow mottle virus: A Threat to genetic Resistance Deployment in West-Central Africa. Phytopathology, 108(2): 299307. DOI: https://doi.org/10.1094/phyto05-17-0190-r.

Issaka S, Basso A, Sorho F, Onasanya A, Haougui A, Sido AY, Aké S, Fargette D, Séré Y. 2012a. Diagnosis and Importance of Rice Yellow Mottle Disease Epidemics in Niger Republic. Journal of Applied Biosciences, 50: 3501-3511.

Issaka S, Onasanya A, Basso A, Sorho F, Haougui A, Sido AY, Ake S, Fargette D, Sere Y. 2012b. Existence of several pathotypes among Rice Yellow Mottle Virus (RYMV) isolates collected in Niger republic. Trends in Applied Sciences Research, 7(1): 32-45. DOI: https://doi.org/10.3923/tasr.2012.32.45.

Issaka S. 2013. La panachure jaune du riz en Afrique de l'Ouest: épidémiologie, distribution, pathogénie et variabilité du Rice Yellow Mottle Virus (RYMV) au Niger. Thèse de Doctorat de l'Université Félix Houphouet Boigny de Cocody, Abidjan-Côte d'Ivoire. 156p.

Kam H, Zongo A, Ouédraogo N, Coulibaly A, Ouédraogo I, Traoré O. 2017. Large effect QTLS involved in resistance to Rice yellow Mottle Virus (RYMV) disease. International Journal of Current Advanced Research, 6(7): 4883-4888. DOI:

http://dx.doi.org/10.24327/ijcar.2017.48 88.0606.

Kam H, Ndjiondjop MN, Ouedraogo N, Laing MD, Ghesquiere A. 2018. Evaluation of rice cultivars for resistance to Rice Yellow Mottle Virus. African Crop Science Journal, 26(1): 49 - 61. DOI: http://dx.doi.org/10.4314/acsj.v26i1.4.

Kouassi NK, N'Guessan P, Albar L, Fauquet CM, Brugidou C. 2005. Distribution and characterization of Rice yellow mottle virus: a threat to African farmers. Plant Dis., 89(2): 124-133. DOI: http://dx.doi.org/10.1094/PD-89-0124. 
Kouassi KN, Chen L, Siré C, Bangratz-Reyser M, Beachy RN, Fauquet CM, Brugidou C. 2006. Expression of rice yellow mottle virus coat protein enhances virus infection in transgenic plants. Arch Virol., 151(11): 2111-2122. DOI: https://doi.org/10.1007/s00705-0060802-3.

Longué RDS, Zinga I, Semballa S, Barro N, Traoré O. 2016. Detection and Serological Characterization of Rice Yellow Mottle Virus in Central African Republic. Agricultural Sciences, 7(12): 911-919. DOI:

http://dx.doi.org/10.4236/as.2016.71208 2

Longué RDS, Pinel-Galzi A, Zinga I, Semballa S, Fargette D, Barro N, Traoré O. 2017. Genetic Diversity and Molecular Epidemiology of Rice Yellow Mottle Virus in Central African Republic. Int. J. Virol., 13(2): 80-89. DOI:

https://dx.doi.org/10.3923/ijv.2017.80.89

Ndikumana I, Gasoré R, Issaka S, Pinel-Galzi A, Onasanya A, Hassani-Mehraban A, Fargette D, Peters D, Séré Y. 2011. Rice yellow mottle virus in rice in Rwanda: first report and evidence of strain circulation. New Disease Reports, 23: 18.

DOI:

http://dx.doi.org/10.5197/j.20440588.2011.023.018.

Ndikumana I, Pinel-Galzi A, Négussié Z, Msolla SN, Njau P, Singh R, Choi IR, Birigimana J, Fargette D, Hébrard E. 2012. First Report of Rice yellow mottle virus on Rice in Burundi. Plant Disease, 96(1): $1230-1230 . \quad$ DOI: https://dx.doi.org/10.1094/PDIS-03-120293-PDN.

Ochola D, Tusiime G. 2011. Survey on incidences and severity of Rice yellow mottle virus disease in Eastern Uganda. International Journal of Plant Pathology, 2(1): 15-25. DOI: http://dx.doi.org/10.3923/ijpp.2011.15.2 5.
Ochola D, Issaka S, Rakotomalala M, PinelGalzi A, Ndikumana I, Hubertf J, Hébrard E, Séré Y, Tusiime G, Fargette D. 2015. Emergence of Rice yellow mottle virus in eastern Uganda: unexpected interplay between strains in East Africa and in Madagascar. Virus Research, 195(2): 64-72. DOI: http://dx.doi.org/10.1016/j.virusres.2014. 09.004 .

Oludare A, Tossou HT, Kini K, Silué D. 2016. Diversity of Rice yellow mottle virus in Benin and Togo and Screening for Resistant Accessions. J. Phytopathol., 164(11-12): 924-935. DOI: http://dx.doi.org/10.1111/jph.12512.

Pidon $\mathrm{H}$, Ghesquière $\mathrm{A}$, Cheron $\mathrm{S}$, Issaka $\mathrm{S}$, Hebrard E, Sabot F, Kolade O, Silué D, Albar L. 2017. Fine mapping of RYMV3: A new resistance gene to Rice yellow mottle virus from Oryza glaberrima. Theor. Appl. Genet., 130(4): 807-818.

DOI: http://dx.doi.org/10.1007/s00122-0172853-0.

Rakotamalala M, Pinel-Galzi A, Albar L, Ghesquière A, Rabenantoandro Y, Ramavovololona P, Fargette D. 2008. Resistance to Rice yellow mottle virus in germplasm in Madagascar. Eur. J. Plant Pathol., 122(12): 277-286. DOI: https://doi.org/10.1007/s10658-0089282-5.

Sarra S, Oevering P, Guindo S, Peters D. 2004. Wind-mediated spread of Rice yellow mottle virus (RYMV) in irrigated rice crops. Plant Pathology, 53(2): 148153.

DOI: https://doi.org/10.1111/j.00320862.2004.00981.x.

Séré Y, Sorho F, Onasanya A, Jobe L, Darboe S, Bojang Y, Touray ML, Pinel-Galzi A, Fargette D. 2008. First report of rice yellow mottle virus in rice in the Gambia. Plant Diseases, 92(2): 316. DOI: https://doi.org/10.1094/PDIS-92-20316B.

Soko DF, Ayolie K, Koffi NBC, Tonessia DC, Sere Y, Kouakou TH, Ake S. 2015. Impact of eight isolates of Rice Yellow 
Mottle Virus (RYMV) from Gagnoa (Côte d'Ivoire) on rice (Oryza sp) cultivars production. Int. J. Biol. Chem. Sci., 9(5): 2459-2467. DOI: http://dx.doi.org/10.4314/ijbcs.v9i5.17.

Sorho F, Pinel A, Traoré O, Bersoult A, Ghesquière A, Hébrard E, Konaté G, Séré Y, Fargette D. 2005. Durability of natural and transgenic resistances in Rice to Rice Yellow Mottle Virus. European Journal of Plant Pathology, 112(4): 49359.

DOI: https://doi.org/10.1007/s10658005-6607-5.

Tall H, Aribi J, Camara S, Pinel-Galzi A, Poulicard N, Fargette D, Hébrard E. 2020. Nearcomplete genome sequences of Rice yellow mottle virus isolates from Senegal. Microbiol Resour Announc., 9(2): $\quad$ e00937-19. DOI: https://doi.org/10.1128/MRA.00937-19.

Thiémélé D, Boisnard A, Ndjiondjop M, Chéron $S$, Séré $Y$, Aké $S$, Ghesquière $A$, Albar L. 2010. Identification of a second major resistance gene to Rice yellow mottle virus, RYMV2, in the African cultivated rice species, $O$. glaberrima. Theor. Appl. Gen., 121(1): 169-179. DOI: https://doi.org/10.1007/s00122010-1300-2.

Traoré O, Sorho F, Pinel A, Abubakar Z, Banwo O, Maley J, Hébrard E, Winter S,
Séré Y, Konaté G, Fargette D. 2005. Processes of diversification and dispersion of Rice yellow mottle virus inferred from large-scale and highresolution phylogeographical studies. Molecular Ecology, 14(7): 20 97-2110. DOI: https://doi.org/10.1111/j.1365294X.2005.02578.x.

Traoré O, Pinel A, Hébrard E, Gumedzoe M, Fargette D, Traoré AS, Konaté G. 2006. Occurrence and frequency of resistancebreaking isolates of Rice yellow mottle virus in the Sudan savannah zone of Africa. Plant Dis., 90(3): 259-263. DOI: https://doi.org/10.1094/PD-90-0259.

Traoré O, Pinel-Galzi A, Sorho F, Sarra S, Rakotomalala M, Sangu E, Kanyeka Z, Séré Y, Konaté G, Fargette D. 2009. A reassessment of the epidemiology of Rice yellow mottle virus following recent advances in field and molecular studies. Virus Res., 141(2): 258-67. DOI: https://doi.org/10.1016/j.virusres.2009.0 1.011.

Traoré O, Pinel-Galzi A, Issaka S, Poulicard N, Aribi J, Aké S. 2010. The adaptation of Rice yellow mottle virus to the eIF(iso) 4G-mediated rice resistance. Virology, 408(1): 103-108. DOI: https://doi.org/10.1016/j.virol.2010.09.0 07. 